\title{
Evaluation of the neuroprotective effects of ozone in an experimental spine injury model
}

\author{
Gokhan Gürkan, MD, ${ }^{1}$ Murat Sayin, MD, ${ }^{1}$ Ceren Kizmazoglu, MD, ${ }^{2}$ \\ Mumin Alper Erdogan, DVM, PhD, ${ }^{3}$ Gurkan Yigitturk, PhD, ${ }^{4}$ Huriye Erbak Yilmaz, MD, ${ }^{5}$ \\ Inan Uzunoglu, MD, ${ }^{1}$ Ismail Kaya, MD, ${ }^{6}$ and Nurullah Yuceer, MD ${ }^{1}$
}

\begin{abstract}
1Department of Neurosurgery, Katip Celebi University Atatürk Training and Research Hospital, Izmir; ${ }^{2}$ Department of Neurosurgery, Dokuz Eylul University Hospital, Izmir; ${ }^{3}$ Department of Physiology, Katip Celebi University, Izmir; ${ }^{4}$ Department of Histology, Sitki Kocman University, Mugla; ${ }^{5}$ Department of Medical Biochemistry, Katip Celebi University Atatürk Training and Research Hospital, Izmir; and ${ }^{6}$ Department of Neurosurgery, Dumlupinar University Kutahya Evliya Celebi Training and Research Hospital, Kutahya, Turkey
\end{abstract}

\begin{abstract}
OBJECTIVE The pathophysiology of spine injury consists of primary and secondary damage mechanisms. The vast majority of treatments aim to prevent or at least stop the progression of secondary neurotoxic events during the acute period. Ozone has been found to have potent antiinflammatory effects, to activate the immune system, and to have a substantial impact on the antioxidant system. In this study the authors aimed to evaluate the neuroprotective effects of ozone and their possible roles in recovery from spine injury, assessed based on biochemical, histological, and neurological parameters using an experimental spine injury model in rats.
\end{abstract}

METHODS The study included 31 female Wistar albino rats. The rats were divided randomly into 5 groups, with 7 rats in each group except the sham group, which contained 3 rats, as follows: group 1 (sham), laminectomy; group 2 (control), laminectomy and spinal trauma with no medical treatment ( $0.5 \mathrm{ml}$ isotonic saline applied 1 hour postsurgery); group 3, single medical treatment with $30 \mathrm{mg} / \mathrm{kg}$ methylprednisolone applied intraperitoneally 1 hour after laminectomy and trauma; group 4, single medical treatment with $60 \mu \mathrm{g} / \mathrm{ml}$ ozone at $0.7 \mathrm{mg} / \mathrm{kg}$ applied intraperitoneally 1 hour after laminectomy and trauma; and group 5, double medical treatment with $30 \mathrm{mg} / \mathrm{kg}$ methylprednisolone and $60 \mu \mathrm{g} / \mathrm{ml}$ ozone at $0.7 \mathrm{mg} / \mathrm{kg}$ applied intraperitoneally 1 hour after laminectomy and trauma. After neurosurgery, neurobehavioral tests were performed in all groups. After 7 days of follow-up, all the rats were killed. Biopsy specimens obtained from trauma sites were examined using $\mathrm{H} \& \mathrm{E}$, cresyl violet, immunohistochemical (anticonnexin-43), and TUNEL staining processes. Levels of interleukin (IL)-1 $\beta$, IL-6, and tumor necrosis factor- $\alpha$ (TNF- $\alpha$ ) and total oxidant status (TOS) and total antioxidant status (TAS) were measured in blood samples.

RESULTS The level of neurobehavioral healing was the highest in the double-treatment group (group 5), and the difference between the groups was significant. The minimum IL-6 level was found in group 5, indicating that the antiinflammatory impact was the most significant in this group $(p=0.01)$. Additionally, ozone was found to reduce oxidant stress more effectively than methylprednisolone $(p=0.03)$. Although methylprednisolone was superior to ozone in terms of the antiinflammatory effect, this effect was greater in group 5 . Nevertheless, the number of neurons in group 5 was close to that of the control group, and the number of apoptotic cells was the least in group $5(p<0.001)$.

CONCLUSIONS In acute spinal injury, the combined application of methylprednisolone and ozone was found to have a greater antiinflammatory effect, hasten clinical recovery, and increase histological recovery compared with methylprednisolone therapy alone. This study showed that this combination therapy of methylprednisolone with the addition of ozone might have a more beneficial effect in the treatment of spinal injury than methylprednisolone therapy alone.

https://thejns.org/doi/abs/10.3171/2020.2.SPINE191439

KEYWORDS ozone treatment; methylprednisolone; experimental spinal cord injury; IL-6; TAS; total oxidant status

ABBREVIATIONS BBB = Basso, Beattie, and Bresnahan; DM = Drummond and Moore; ELISA = enzyme-linked immunosorbent assay; IL = interleukin; IPA = inclined plane angle; IPT = inclined plane test; TAS = total antioxidant status; TNF- $\alpha=$ tumor necrosis factor $-\alpha$; TOS = total oxidant status.

SUBMITTED December 3, 2019 ACCEPTED February 20, 2020.

INCLUDE WHEN CITING Published online May 15, 2020; DOI: 10.3171/2020.2.SPINE191439. 
$\mathrm{T}$ HE spinal cord cannot renew itself. Thus, permanent damage occurs in patients with spinal cord injury. ${ }^{1,2}$ Two mechanisms in spinal cord injury increase the risk of damage, ${ }^{3,4}$ primary mechanical injury and secondary ischemic injury. Mechanical damage occurs during the incident, damaging the nerves, the spinal cord itself, and/or spinal cord structures. ${ }^{5}$ Secondary injury is caused by metabolic and biochemical factors and occurs within the hours following primary injury. ${ }^{6,7}$ Ischemia resulting in insufficient perfusion during the early postinjury period is one of the most critical factors that leads to secondary damage. ${ }^{4,8}$

Along with secondary damage, calcium, stimulatory amino acids, free oxygen radicals, and many chemicals, such as tumor necrosis factor- $\alpha$ (TNF- $\alpha)$, interleukin (IL) $-1 \beta$, IL-6, and IL-8, are released. ${ }^{4,9}$ According to Fehlings and Tator, ischemia is the basis of secondary injury, but the process is treatable and preventable. ${ }^{1}$ The primary objective of all experimental and clinical studies regarding traumatic spinal cord injury is the reduction of secondary damage.

The neuroprotective effects of many pharmacological agents, such as methylprednisolone, melatonin, erythropoietin, magnesium, mexiletine, naloxone, infliximab, clotrimazole, lamotrigine, lazaroids, neutralizing antibodies, and hyperbaric oxygen, have been investigated in experimental spinal cord injury. ${ }^{10-15}$ Among these agents, only methylprednisolone has been introduced into clinical use, and it has been shown to have positive effects after the injury. However, despite the positive effects of methylprednisolone on parenchymal damage, the drug has been shown not to affect functional recovery. ${ }^{16}$

Proinflammatory cytokines such as IL-1 $\beta$, IL-6, and TNF- $\alpha$ increase within a few hours after spinal cord injury. Recent studies have shown that these cytokines are directly related to permanent motor dysfunction and histopathological damage. ${ }^{17-19}$

Oxidative stress plays a significant role in the progression of spinal cord lesions after the primary injury. Neuronal tissues, such as the spinal cord, are highly sensitive to oxidative stress as they contain a large amount of lipids.
The total antioxidant status (TAS) represents the antioxidant defense level of an organism, and the total oxidant status (TOS) represents the oxidant load. These two parameters are reliable indicators of oxidative stress when used together. ${ }^{20,21}$

Ozone $\left(\mathrm{O}_{3}\right)$ is a gaseous molecule that consists of 3 oxygen atoms. ${ }^{22}$ Ozone has been reported to be effective for treating arterial circulatory disorders, burns, proctitis, colitis, and wounds and as an additional treatment in carcinogenic cases, geriatric problems, rheumatic diseases, age-related macular degeneration, arthritis, and ischemic and infectious (herpes and hepatitis) diseases. Ozone is particularly useful in the treatment of infective wounds due to complications of diabetes. ${ }^{23}$ Chronic oxidative stress and antioxidant insufficiency are common factors in these diseases. Ozone plays a role in the activation of red blood cell metabolism and in the release of essential cytokines such as interferon and IL by activating immune cells. It also induces antioxidant activation. ${ }^{24,25}$

In this study we aimed to investigate the neuroprotective effects in the traumatized spinal cord of ozone, which is commonly used to treat chronic oxidative stress and diseases related to antioxidant insufficiency.

\section{Methods \\ Experimental Groups}

The surgical procedures in this study were carried out in the research laboratories of the Izmir Dokuz Eylul University Hospital for experimental animals with the permission of the university's experimental animals ethics committee (reference number 54/2016). Thirty-one female Wistar albino rats (3 rats in the sham group and 7 rats in each of the other 4 groups) were used. The rats in each group weighed 250-300 $\mathrm{g}$ and were randomly assigned to 1 of 5 groups (Fig. 1) - group 1: sham, laminectomy only; group 2: controls, laminectomy and trauma performed and $0.5 \mathrm{ml}$ isotonic saline applied 1 hour after trauma; group 3: methylprednisolone treatment only, $30 \mathrm{mg} / \mathrm{kg}$ methylprednisolone administered intraperitoneally 1 hour after laminectomy and trauma; group 4: ozone treatment

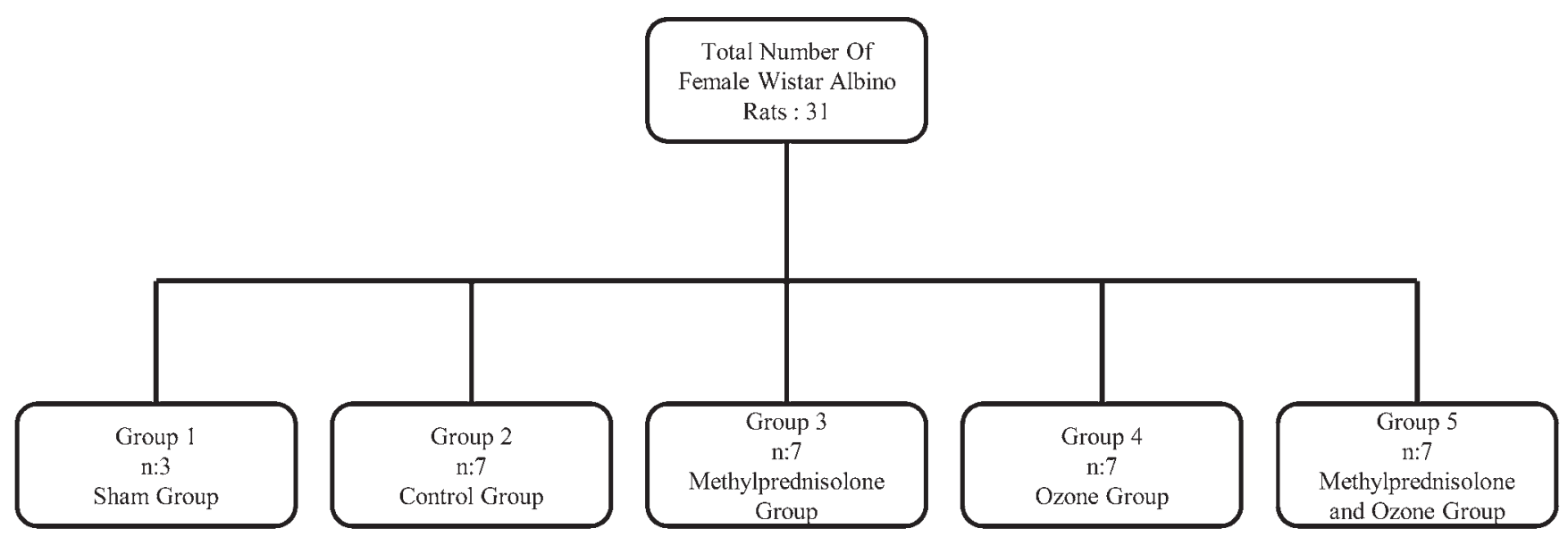

FIG. 1. Design of the study. 


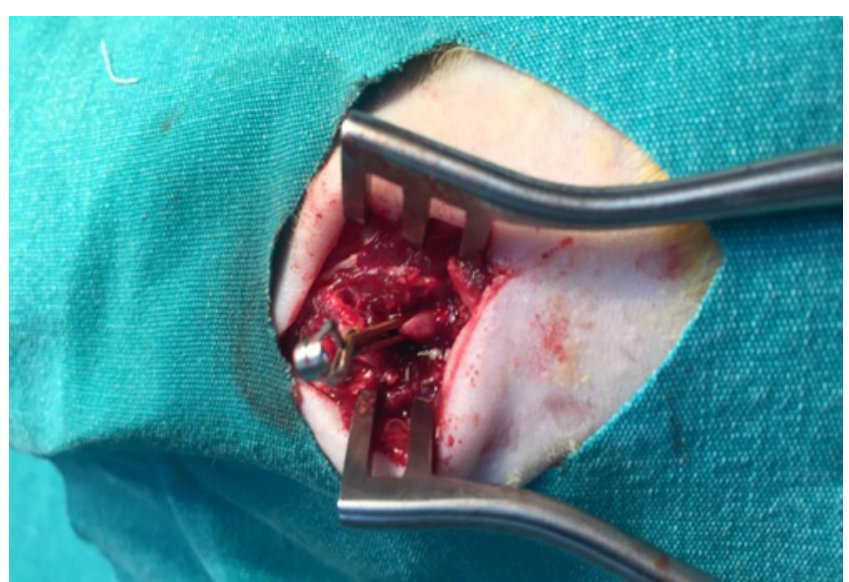

FIG. 2. Application of the Tator and Rivlin model. Figure is available in color online only.

only, $60 \mu \mathrm{g} / \mathrm{ml}$ ozone administered at $0.7 \mathrm{mg} / \mathrm{kg}$ intraperitoneally 1 hour after laminectomy and trauma; and group 5: double treatment with methylprednisolone and ozone combined, $30 \mathrm{mg} / \mathrm{kg}$ methylprednisolone $+60 \mu \mathrm{g} / \mathrm{ml}$ ozone administered at $0.7 \mathrm{mg} / \mathrm{kg}$ intraperitoneally 1 hour after laminectomy and trauma.

The medication doses administered were determined in line with previously reported studies that included similar animal experiments. ${ }^{11-14,16-18}$

\section{Anesthesia}

All rats were injected intraperitoneally with $50 \mathrm{mg} / \mathrm{kg}$ ketamine $\mathrm{HCl}$ (Ketalar, Pfizer) and $5 \mathrm{mg} / \mathrm{kg}$ xylazine (Rompun, Bayer) for general anesthesia.

\section{Surgery}

All rats, after receiving anesthesia, were fixed on individual surgical boards in the prone position. The back area of all animals was shaved under general anesthesia, and povidone iodine (Batticon, Adeka) was used as a local antiseptic. A midline incision was made at the level of T5-12 with reference to the interscapular distance of the rat in prone position. The skin and subcutaneous tissues were dissected, and the paravertebral muscle fascia was opened. The muscles were then stripped laterally by blunt dissection. The T7-10 lamina was exposed, and a total laminectomy was performed. During these procedures, the dura mater was preserved.

In group 1 rats, no additional procedures other than laminectomy were performed. In group 2, spinal trauma was created by applying a clip extradurally for 1 minute following the laminectomy, and $0.5 \mathrm{ml}$ physiological saline solution was administered intraperitoneally after 1 hour. In group 3, $30 \mathrm{mg} / \mathrm{kg}$ methylprednisolone was administered intraperitoneally 1 hour after laminectomy and spinal trauma. In group 4, $60 \mu \mathrm{g} / \mathrm{ml}$ ozone at $0.7 \mathrm{mg} / \mathrm{kg}$ was administered intraperitoneally 1 hour after laminectomy and trauma. In group 5,30 mg/kg methylprednisolone + $60 \mu \mathrm{g} / \mathrm{ml}$ ozone at $0.7 \mathrm{mg} / \mathrm{kg}$ was administered intraperitoneally 1 hour after laminectomy and spinal trauma. To ensure that the spinal trauma was standardized, a Yaşargil

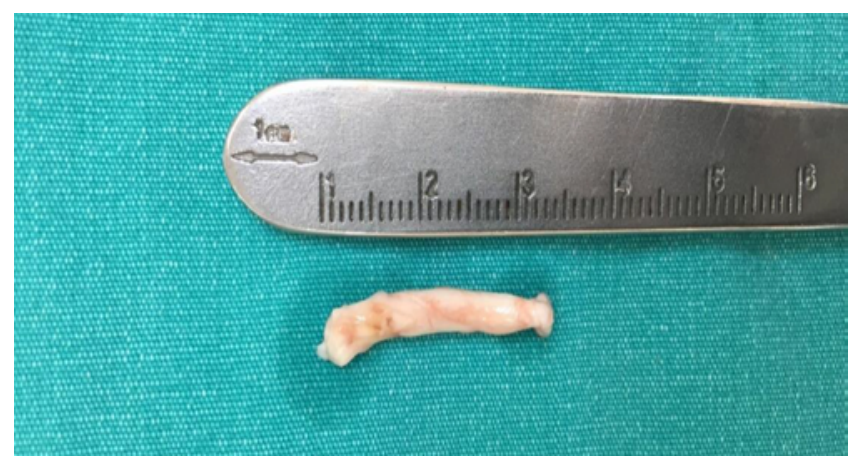

FIG. 3. Dissected spinal cord of a rat. Figure is available in color online only.

FE 721 aneurysm clip (Aesculap) that exerts $70 \mathrm{~g}$ of force was applied to wrap around the dural and spinal cord for 1 minute in accordance with the model developed by Tator and Rivlin ${ }^{1}$ (Fig. 2). Following hemostasis, the muscles and incision were appropriately closed. All rats subjected to spinal trauma (groups 2-5) were found to be paraplegic after the procedure. All rats were placed in their cages from the postoperative period until the end of the recovery period and allowed free access to water and food. During the follow-up period, the bladders of the subjects were emptied manually by bladder massages performed twice a day. Intraperitoneal cefazolin sodium at $40 \mathrm{mg} / \mathrm{kg} / \mathrm{day}$ (Cefamezin, Eczacıbaşı Pharmaceutical Industries) was administered to all rats to prevent surgical field and urinary tract infection during the first 3 days postoperatively. The dressings of the subjects were changed regularly during the follow-up period.

Behavioral tests and functional improvements were evaluated during the 1-week follow-up period after surgery. Rats were anesthetized before being killed, and blood samples for biochemical analysis were taken from the heart under sterile conditions. Afterward, the surgical wound area was reopened with the rats in the prone position, and a 2-cm sample was dissected from the center of the damaged spinal cord and sent for histopathological examination (Fig. 3).

\section{Behavioral Tests and Evaluation of Functional Recovery Inclined Plane Test}

Functional recovery in rats was evaluated using the inclined plane test (IPT), which is commonly used in experimental spinal cord injury studies. ${ }^{4}$ In this method, after being placed on a flat table, the subject is motivated with food, and the subject's climbing behavior is observed. The table is initially parallel to the ground, and the angle gradually increases during the test. The highest angle at which the subject can stand on the table without falling for 5 seconds is considered the inclined plane angle (IPA) for the subject. The IPT was administered to all rats on days $1,3,5$, and 7 after spinal trauma, and the scores were recorded.

\section{Basso, Beattie, and Bresnahan Scale}

The Basso, Beattie, and Bresnahan (BBB) scale, which 
is frequently used by researchers for the measurement of behavioral outcomes after spinal cord injury treatments, was developed in 1995 by Basso et al. ${ }^{26}$

To determine the BBB score, a wide range of locomotor functions is evaluated. Scores range from 0 to 21 points. The scoring system includes information varying from the absence of any movement in the hindlimbs to the number of joints involved in the movement, coordination of the movements, and finally, full-body stability and the tail being in the air. ${ }^{26}$

All rats underwent BBB scoring on days 1, 3, 5, and 7 after spinal trauma. Since follow-up was performed only during the acute period after spinal cord injury in our study, only point scores from 0 to 7 , which are used in the $\mathrm{BBB}$ scale to evaluate the early phase of recovery, were taken into consideration.

\section{Modified Tarlov Scale}

Open-field behaviors were evaluated and scored by using the modified Tarlov rating scale as follows: ${ }^{27}$

1. No movement

2. Minimal movement of the hind legs but cannot stand on the hind legs

3. Can stand on its legs but cannot walk

4. Can walk with some spasticity, or there is incoordination in the hindlimbs

5. Normal motor movement

\section{Drummond and Moore Criteria}

To determine the functional improvements in all rats, posttraumatic motor examinations were performed on days 1, 3, 5, and 7 using the Drummond and Moore (DM) scale as follows: ${ }^{14}$

0 . Paraplegia, no motor function in the lower extremities

1. Weak motor function in the lower extremities

2. Intermediate motor function in the lower extremities, but cannot pull the extremities under the body

3. Strong motor function in the lower extremities and can pull the extremities under the body

4. Normal motor function

\section{Biochemical Analysis}

Plasma TAS and TOS measurements of the rats in the sham and trauma groups were performed spectrophotometrically using an Abbott C16000 device with a commercially available measurement kit.

The serum concentrations $(\mathrm{pg} / \mathrm{ml})$ of inflammatory mediators (IL-1 $\beta$, IL-6, and TNF- $\alpha$ ) were calculated. For the enzyme-linked immunosorbent assay (ELISA), rat IL$1 \beta$, IL-6, and TNF- $\alpha$ kits were used with a Biotek semiautomatic ELISA device.

\section{Histopathological Evaluation}

At the end of day 7 after spinal injury, anesthesia was administered by injection of a solution of ketamine and xylazine, and blood samples were taken from the heart for measurements of serum TAS, TOS, IL-1 $\beta$, IL-6, and TNF- $\alpha$. To obtain the damaged tissue, skin sutures were removed, and the paravertebral muscles were pulled aside. A 2-cm-long cord segment $(1 \mathrm{~cm}$ rostral and $1 \mathrm{~cm}$ caudal to the trauma zone in the center) was harvested. All samples were fixed in $10 \%$ neutral buffered formalin and embedded in paraffin. Sections with a thickness of 5 $\mu \mathrm{m}$ were cut using a microtome. The tissue sections were stained with $\mathrm{H} \& \mathrm{E}$ for assessment using a semiquantitative rating scale (Table 1). Cresyl violet was used for neuron counting. Anticonnexin-43, which was reported to show the immunopositive reaction in motor neurons, fibrous and protoplasmic astrocytes, and ependymal cells in the medulla spinalis tissue, was used for immunohistochemical analysis. The TUNEL technique was used for apoptotic cell counting. The evaluations were performed using an Olympus BX-51 light microscope and imaging device.

\section{Statistical Analysis}

The SPSS V21.0 (IBM Corp.) program was used for statistical analysis. Descriptive statistics are presented as numbers and percentages for categorical variables and as mean values and standard deviations for numerical variables. For numerical variables, the Kruskal-Wallis test was used when a normal distribution was not identified in multiple independent group comparisons, and the MannWhitney U-test was used when a normal distribution was not identified in binary independent group comparisons. The chi-square test was used for categorical variables, and Spearman's rho test was used for correlations in which a normal distribution was not found in the correlation between numerical data. The statistical significance level was accepted as a p value less than 0.05 .

\section{Results}

\section{Results of Behavioral Tests and Functional Improvement Assessments}

The results of the assessments of the groups performed with the IPT, BBB, modified Tarlov, and DM methods differed among themselves on days 1, 3,5, and 7 after spinal injury. In Table 2, the significant $\mathrm{p}$ values of the comparisons of the results between days for each group are shown.

Although a significant difference in the IPA scores was found between days 1 and 3 in group $3(p=0.008)$, the BBB scores for these days did not significantly differ. A significant difference was found between the day 1 and 7 scores for the modified Tarlov scale $(p=0.020)$, but there was no significant difference between these days for the DM test results.

The differences between the scores for days 1 and 7 after spinal injury were statistically significant $(\mathrm{p}=0.015$ and $p=0.034$ ) for the IPA and BBB scores found in group 4 , and the difference between days 1 and 5 was not significant $(\mathrm{p}=0.025)$ for the modified Tarlov scale. In the DM test, no significant difference was found between the scores for these days.

In group 5, in which both treatments were administered, significant differences were found between days 1 and 7 for all of the tests $(p=0.015, p=0.014, p=0.011$, and $\mathrm{p}=0.011$ ).

\section{Biochemical Results}

The levels of IL-1 $\beta$, IL-6, and TNF- $\alpha$, which are 
TABLE 1. Semiquantitative rating scale for histopathological assessment

\begin{tabular}{cc}
\hline Histological Description & Score \\
\hline Edema & 0 \\
\hline No edematous tissue & 1 \\
\hline Minimal edema & 2 \\
\hline Moderate edema & 3 \\
\hline Severe edema & \\
\hline Tissue necrosis & 0 \\
\hline No necrotic tissue & 1 \\
\hline $1 \%-10 \%$ necrosis & 2 \\
\hline$>10 \%-25 \%$ necrosis & 3 \\
\hline$>25 \%$ necrosis & \\
\hline Hemorrhage & 0 \\
\hline No hemorrhage & 1 \\
\hline Minimal hemorrhage & 2 \\
\hline Moderate hemorrhage & 3 \\
\hline Severe hemorrhage & 0 \\
\hline Inflammation & 1 \\
\hline No inflammatory cells & 2 \\
\hline Several focal cells & 3 \\
\hline Excessive number of cells & \\
\hline Abscess formation & \\
\hline
\end{tabular}

markers of inflammation, were measured biochemically. Compared to the sham group, a significant increase was observed in the spinal trauma groups for all 3 mediators. There was no significant difference in these markers between groups 3 and 4, but the IL-6 levels were significantly lower in group 5 than in group $4(\mathrm{p}=0.010)$.

The TOS and TAS levels were evaluated in all groups to determine the oxidant and antioxidant status. The TOS level in group 4 was significantly lower $(p=0.030)$ than that in group 3, but this difference was not found in the TAS levels. The TAS level in group 5 was significantly higher $(\mathrm{p}=0.040)$ than that in group 4.

\section{Histopathological Evaluation Results}

When the total scores for edema, hemorrhage, necrosis, and inflammation were assessed histologically, there was a significant increase in group 2 compared with the sham group, whereas no significant difference was found between groups 3 and $4(\mathrm{p}=0.89)$. Compared to groups 3 and 4, there was a significant decrease in the score for group $5(\mathrm{p}=0.018)$.

The numbers of neurons were examined in all groups using cresyl violet dye, and the results for group 5 were closest to those for the sham group. Therefore, the group 5 rats treated with both methylprednisolone and ozone had the best outcome. No significant difference was found between the single-treatment groups 3 and 4 . In the evaluation of apoptosis using the TUNEL technique, the number

TABLE 2. Comparison of $p$ values for behavior test scores and functional improvement assessment results between days for each group with spinal trauma (groups 2-5)

\begin{tabular}{|c|c|c|c|c|c|c|}
\hline \multirow[b]{2}{*}{ Behavior Test } & \multicolumn{6}{|c|}{ Score $p$ Value } \\
\hline & Days 1-3 & Days $1-5$ & Days 1-7 & Days 3-5 & Days 3-7 & Days $5-7$ \\
\hline \multicolumn{7}{|l|}{ Group 2} \\
\hline IPT & - & - & 0.046 & - & 0.014 & 0.046 \\
\hline BBB & 0.039 & - & 0.014 & - & 0.014 & 0.014 \\
\hline Modified Tarlov scale & 0.046 & 0.046 & 0.023 & - & 0.046 & 0.046 \\
\hline $\mathrm{DM}$ & - & - & 0.014 & - & 0.046 & - \\
\hline \multicolumn{7}{|l|}{ Group 3} \\
\hline IPT & 0.008 & - & - & - & 0.014 & 0.02 \\
\hline BBB & - & - & - & - & - & - \\
\hline Modified Tarlov scale & - & - & 0.02 & - & 0.046 & 0.025 \\
\hline $\mathrm{DM}$ & - & - & - & - & - & - \\
\hline \multicolumn{7}{|l|}{ Group 4} \\
\hline IPT & - & - & 0.015 & - & 0.011 & 0.015 \\
\hline BBB & - & - & 0.034 & - & - & - \\
\hline Modified Tarlov scale & - & 0.025 & - & - & - & - \\
\hline DM & - & - & - & - & - & - \\
\hline \multicolumn{7}{|l|}{ Group 5} \\
\hline IPT & 0.014 & 0.014 & 0.015 & - & 0.014 & 0.014 \\
\hline BBB & 0.014 & 0.014 & 0.014 & - & 0.038 & 0.015 \\
\hline Modified Tarlov scale & 0.015 & - & 0.011 & - & 0.046 & 0.038 \\
\hline $\mathrm{DM}$ & - & - & 0.011 & - & 0.034 & 0.025 \\
\hline
\end{tabular}




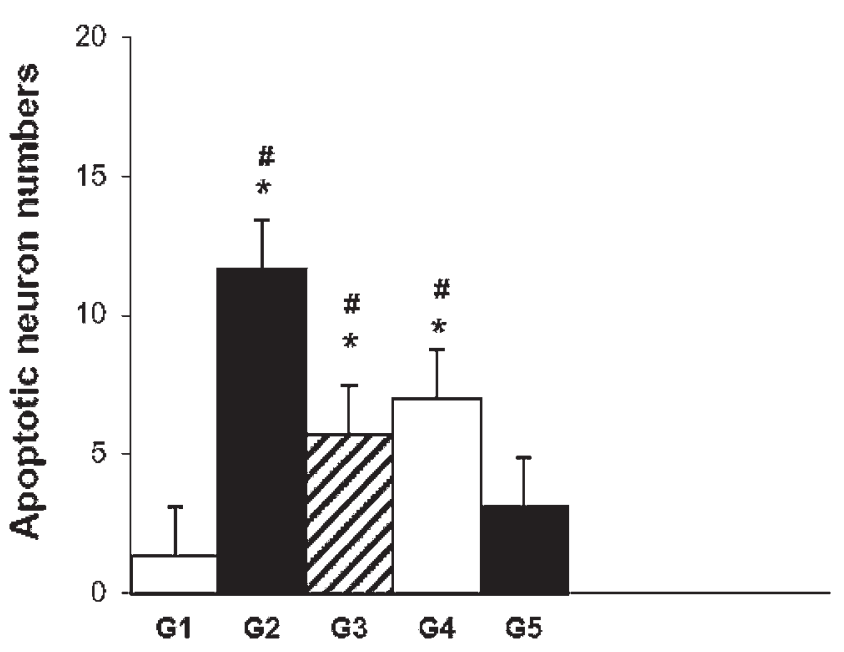

FIG. 4. Statistical evaluation of the numbers of TUNEL-positive (apoptotic) neurons in the 5 groups. ${ }^{*} p<0.001$ compared with group 1 ; $\# p<$ 0.001 compared with group 5 .

of positive cells (Fig. 4) in group 5 was closest to that of the sham group, which had the fewest positive cells, followed by groups 3 and 4, respectively. Further, group 5 had the highest number of anticonnexin-43-positive stained cells (Fig. 5). There was no significant difference between groups 3 and 4 .

\section{Discussion}

The high incidence of spinal cord trauma, especially in the young and productive population, is a health problem that continues to require substantial attention today because of the physical, psychosocial, and economic damage from these injuries, which frequently cause permanent and irreversible motor deficits and continued decrease in the quality of life of the patients after injury. ${ }^{28}$ These deficits occur via primary and secondary damage mechanisms. In particular, the secondary damage processes include edema, inflammation, free radical formation, and lipid peroxidation. Treatment regimens should therefore be adjusted accordingly. ${ }^{29}$ Ozone therapy, previously used just for disinfection and sterilization, is currently being explored as a treatment method in many diseases for which currently available medical treatment is insufficient, and useful results have been reported..$^{30}$ Medical ozone treatment has been found to accelerate decongestion and cause edema reabsorption. ${ }^{31}$

In a study by Yamanel et al., ozone therapy reduced systemic inflammatory processes and decreased oxidative stress in tissues. ${ }^{32}$ There have been many studies on the antiinflammatory activity of ozone. In a study by Uysal et al. using an experimental rat model of uterine adhesion, ozone therapy prevented postoperative uterine adhesions by modulating the TNF- $\alpha$ levels and the oxidative/antioxidative status.33

We investigated the effects of methylprednisolone, ozone, and a combination of both treatments on clinical, biochemical, and histopathological markers in rats with spinal cord injury. During the follow-up period, the pa-

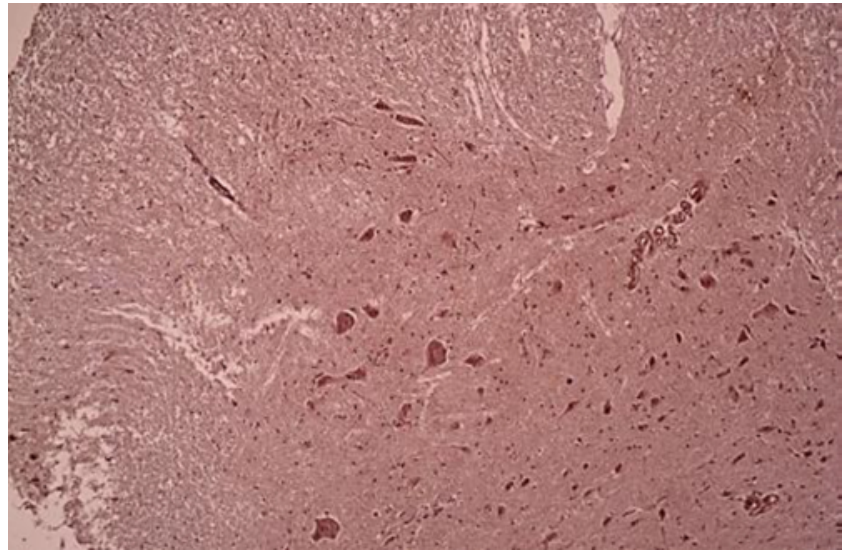

FIG. 5. Anticonnexin-43 immune staining sample for group 5, which had the highest number of anticonnexin-43-positive stained cells. Original magnification $\times 20$. Figure is available in color online only.

tients who received combination therapy (group 5) were the closest to the sham group (group 1) with regard to clinical improvement, followed by the group that received methylprednisolone treatment only (group 3) and then the group that received ozone therapy only (group 4). The clinical response was the best in the double-treatment group receiving a combination of methylprednisolone and ozone (group 5).

There were no significant differences between the methylprednisolone group and the ozone group in terms of the levels of IL-1 $\beta$, IL- 6 , and TNF- $\alpha$, which are biochemical markers of inflammation. Compared to the group that received ozone treatment alone, the IL-6 levels were significantly lower in the double-treatment group. This observation was interpreted to mean that the addition of methylprednisolone to ozone increased the antiinflammatory power of the former.

The TOS and TAS levels were studied in all groups to determine the status of oxidants and antioxidants. In the group that received ozone therapy only (group 4), the TOS level was significantly lower than the level in the methylprednisolone-only group (group 3), but this difference was not observed for the TAS level. The level of TAS in the double-treatment group (group 5) was significantly higher than that in the ozone-only treatment group. This finding suggested that ozone reduces oxidant stress more effectively than methylprednisolone and increases the TAS level when combined with methylprednisolone. However, despite the beneficial effects of methylprednisolone on the oxidant and antioxidant balance, clinical superiority was not observed in the methylprednisolone-only group, indicating that the results obtained in the ozone group were governed by many different mechanisms.

Although methylprednisolone and ozone have long been known to show antiinflammatory effects, methylprednisolone was found to be superior to ozone in reducing edema and inflammation detected in histopathological analyses. This superiority was greater in the combinedtherapy group.

The number of neurons in the spinal cord sections obtained from all groups was examined with cresyl violet 
staining and was found to be highest in the double-treatment group (group 5). The TUNEL results also showed that double treatment was more effective in preventing apoptosis. Further, the double-treatment group had the most anticonnexin-43-positive cells. There was no significant difference between the methylprednisolone-only and ozone-only groups for these TUNEL results.

In a study that investigated the effects of $\alpha$-lipoic acid and methylprednisolone in a model of experimental spinal cord injury, the IPT was performed 24 hours after the trauma. The results showed that the IPAs increased significantly in the groups in which methylprednisolone and $\alpha$-lipoic acid were administered compared to the angles in the trauma group. ${ }^{34}$ In our study, the double-treatment group had better IPT results than the single-treatment groups on days 5 and 7.

In a study by Yaman et al. examining the effects of hyperbaric oxygen therapy in an experimental spinal cord injury model, the motor powers of the subjects were compared using the DM scale. The scores were better in the treatment group than in the spinal cord injury and sham groups. ${ }^{14}$ In our study, the DM scores for the double-treatment group were higher than the scores for the singletreatment groups.

In a study conducted by Kahveci et al., who examined the effects of methylprednisolone and rosuvastatin in experimental spinal cord injury-induced rats, the BBB scores for treatment with each drug were superior to those for sham treatment but not superior to each other. ${ }^{35}$ Similar results were also obtained in our study, but the BBB scores in group 5, the double-treatment group, were higher than the scores in the other groups.

In research performed by Celik et al. using rats with experimental spinal cord injury, there were significant increases in malondialdehyde, IL-1 $\beta$, IL- 6 , and TNF- $\alpha$ levels in the spinal cord injury group compared to the control group. In all 3 groups treated with adalimumab and/ or methylprednisolone, the levels of these 4 inflammatory molecules were significantly decreased compared to the levels in the spinal cord injury group. Additionally, the IL-1 $\beta$, IL-6, and TNF- $\alpha$ levels were significantly lower in the group administered both adalimumab and methylprednisolone than in the single-treatment group, demonstrating that the combination increased the antiinflammatory power of the treatment. ${ }^{36}$ In our study, IL-6 levels were significantly lower in the double-treatment group (group 5 ) than in the ozone-only treatment group (group 4). However, the IL-1 $\beta$ and TNF- $\alpha$ levels did not differ between these groups. No inflammatory cytokine levels were significantly different between the methylprednisolone and ozone single-treatment groups. The lack of significant differences in the levels of IL-1 $\beta$ and TNF- $\alpha$ between the double-treatment group and the ozone-only treatment group was attributed to the similarity of the antioxidant effects of ozone and methylprednisolone in spinal cord injury, regardless of clinical improvement. In other words, ozone, despite lacking the ability to correct the histopathology and the clinical progress of the injury, has antioxidant activity independent of these effects.

In a study by Aras et al., who examined the effects of minocycline in experimental spinal cord injury, the TAS and TOS levels were significantly lower in the minocycline group than in the spinal cord injury group. ${ }^{37}$ In the present study, the TAS and TOS levels in spinal cord injury were both elevated, and the TAS level was predicted to increase as an endogenous compensatory mechanism due to the trauma. We found that the TAS levels were lowest in the ozone-only treatment group (group 4) and highest in the double-treatment group (group 5), whereas the TOS levels were highest in the methylprednisolone-only group (group 3) and lowest in the double-treatment group. The TAS levels in the double-treatment group were significantly higher than those in the ozone-only group, but no significant differences in TAS levels were found in these groups compared to the methylprednisolone-only group.

The TOS level was significantly higher in the methylprednisolone-only group than in the ozone-only and double-treatment groups. The observation of the lowest TOS level in the double-treatment group indicates that ozone and methylprednisolone potentiate each other in reducing oxidant stress. However, the TAS level was higher in the double-treatment group than in the ozone-only group, but not higher than that in the methylprednisolone-only group. This suggests that the main component that increases antioxidant capacity is methylprednisolone. In addition, as reported in the study by Aras et al., TAS levels are affected more rapidly because of endogenous compensatory mechanisms and seem to be affected by many intracellular mechanisms. ${ }^{37}$

In research conducted by Celik et al., who examined the neuroprotective effects of adalimumab and methylprednisolone in a model of experimental spinal cord injury, the total score was significantly lower in the group that received both adalimumab and methylprednisolone than in the groups that received the drugs individually, similar to our findings. When Celik et al. examined their results in detail, there were no significant differences in the edema and bleeding scores in the treatment groups, but necrosis and inflammation were significantly lower in all treatment groups. ${ }^{36}$ In the present study, when edema, bleeding, necrosis, and inflammation were evaluated separately, the highest scores were found in the rats with spinal cord injury and no medical treatment (group 2), and the lowest scores were found in the double-treatment group (group 5 ). The methylprednisolone-only group (group 3) had less edema and necrosis but more bleeding and inflammation than the ozone-only group (group 4).

Spinal cord injury becomes more complicated secondary to the inflammatory response of the natural immune system to trauma. Previous studies have shown that excessive release of ATP from the peritraumatic site contributes to the inflammatory response to spinal cord injury by activating 7 low-affinity $\mathrm{P} 2 \mathrm{X}$ receptor subunits. However, connexin-43 is also a tight-junction protein and therefore plays an essential role in maintaining tissue integrity. ${ }^{38}$

Because connexin hemichannels are essential pathways for astrocytic ATP release, Huang et al. investigated the effects of deletions in the connexins of astrocytes on posttraumatic ATP release. In 2 groups of rats that had fallen from a height, immunofluorescence microscopy revealed that compared with normal rats, rats with connexin-43 deletion showed a significant reduction in ATP release. 
In addition, in rats with connexin-43 deficiency, less astrogliosis and microglia activation, faster motor recovery, and smaller traumatic lesions were observed. These findings were interpreted to mean that the semichannels of astrocytic connexin-43 exacerbate the secondary injury by increasing posttraumatic ATP release. ${ }^{38}$ In our study, anticonnexin-43 was highly positive in the control (sham) group (group 1), and immune positivity closest to this level was found in the double-treatment group (group 5). Less positive immune staining was detected in both single-treatment groups (groups 3 and 4), and even less was detected in the untreated spinal cord injury group (group 2). According to this result, even if the anticonnexin-43 level in the double-treatment group was not the closest to that of the control group despite better histological recovery, the scoring system, which enables evaluation of tissue morphology, indicates that the tissue structure and cellular integrity were better protected in the double-treatment group.

In a study performed by Zhou et al. to investigate the effects of calcitriol in a model of experimental spinal cord injury, immunohistochemical specimens with apoptosis in histological sections showed negative TUNEL staining in the control group and diffuse positive TUNEL staining in the spinal cord injury group. The TUNEL positivity rate decreased in the calcitriol group. ${ }^{39}$ In our study, the double-treatment group (group 5) was closest to the control group with regard to the level of TUNEL staining in the histological sections. This group was followed in decreasing order of staining level by the methylprednisolone-only and ozone-only treatment groups (groups 3 and 4 , respectively) and the untreated spinal cord injury group (group 2). These results confirmed that coadministration of the treatments better prevented apoptosis than singletreatment administration.

\section{Study Limitations}

There are several limitations to this study. Because this was an experimental animal study, the numbers and types of animals used were determined by the ethics committee. The results of the study might have had more variability if more or different types of animals were used. In addition, we administered the medicines intraperitoneally and caused spinal trauma to the animals. These procedures may have caused minor hemorrhages, and we cannot determine if these minor hemorrhages altered the results of our study.

\section{Conclusions}

In our study of rats with spinal cord injury, rats treated with ozone together with methylprednisolone demonstrated increased antiinflammatory effects, accelerated clinical improvement, and improved histological recovery compared with rats given methylprednisolone treatment alone. This study showed that double treatment with ozone and methylprednisolone might be more useful for spinal cord injury than treatment with methylprednisolone alone. However, more extensive studies are needed before ozone can be considered a treatment option for patients with spinal cord trauma.

\section{References}

1. Fehlings MG, Tator $\mathrm{CH}$. An evidence-based review of decompressive surgery in acute spinal cord injury: rationale, indications, and timing based on experimental and clinical studies. J Neurosurg. 1999;91(1)(suppl):1-11.

2. Horner PJ, Gage FH. Regenerating the damaged central nervous system. Nature. 2000;407(6807):963-970.

3. Mcilvoy LH. The effect of hypothermia and hyperthermia on acute brain injury. AACN Clin Issues. 2005;16(4):488-500.

4. Tator $\mathrm{CH}$, Fehlings MG. Review of the secondary injury theory of acute spinal cord trauma with emphasis on vascular mechanisms. J Neurosurg. 1991;75(1):15-26.

5. Cho Y, Ueda T, Mori A, et al. Protective use of $N$-methyl-Daspartate receptor antagonists as a spinoplegia against excitatory amino acid neurotoxicity. J Vasc Surg. 2005;42(4):765771.

6. Fehlings MG, Sekhon LH, Tator C. The role and timing of decompression in acute spinal cord injury: What do we know? What should we do? Spine (Phila Pa 1976). 2001;26(24)(suppl):S101-S110.

7. Kwon BK, Oxland TR, Tetzlaff W. Animal models used in spinal cord regeneration research. Spine (Phila Pa 1976). 2002;27(14):1504-1510.

8. Feiner JR, Bickler PE, Estrada S, et al. Mild hypothermia, but not propofol, is neuroprotective in organotypic hippocampal cultures. Anesth Analg. 2005;100(1):215-225.

9. Faden AI, Simon RP. A potential role for excitotoxins in the pathophysiology of spinal cord injury. Ann Neurol. 1988;23(6):623-626.

10. İldan F, Polat S, Öner A, et al. Effects of naloxone on sodium- and potassium-activated and magnesium-dependent adenosine-5'-triphosphatase activity and lipid peroxidation and early ultrastructural findings after experimental spinal cord injury. Neurosurgery. 1995;36(4):797-805.

11. Kurt G, Ergün E, Cemil B, et al. Neuroprotective effects of infliximab in experimental spinal cord injury. Surg Neurol. 2009;71(3):332-336.

12. Tufan K, Oztanir N, Ofluoglu E, et al. Ultrastructure protection and attenuation of lipid peroxidation after blockade of presynaptic release of glutamate by lamotrigine in experimental spinal cord injury. Neurosurg Focus. 2008;25(5):E6.

13. Usul H, Arslan E, Cansever T, et al. Effects of clotrimazole on experimental spinal cord ischemia/reperfusion injury in rats. Spine (Phila Pa 1976). 2008;33(26):2863-2867.

14. Yaman O, Yaman B, Aydın F, et al. Hyperbaric oxygen treatment in the experimental spinal cord injury model. Spine $J$. 2014;14(9):2184-2194.

15. Yu Y, Matsuyama Y, Yanase M, et al. Effects of hyperbaric oxygen on GDNF expression and apoptosis in spinal cord injury. Neuroreport. 2004;15(15):2369-2373.

16. Sayer FT, Kronvall E, Nilsson OG. Methylprednisolone treatment in acute spinal cord injury: the myth challenged through a structured analysis of published literature. Spine J. 2006;6(3):335-343.

17. Gong G, Yuan LB, Hu L, et al. Glycyrrhizin attenuates rat ischemic spinal cord injury by suppressing inflammatory cytokines and HMGB1. Acta Pharmacol Sin. 2012;33(1):11-18.

18. Hasturk A, Atalay B, Calisaneller T, et al. Analysis of serum pro-inflammatory cytokine levels after rat spinal cord ische$\mathrm{mia} /$ reperfusion injury and correlation with tissue damage. Turk Neurosurg. 2009;19(4):353-359.

19. Smith PD, Puskas F, Meng X, et al. The evolution of chemokine release supports a bimodal mechanism of spinal cord ischemia and reperfusion injury. Circulation. 2012;126(11) (suppl 1):S110-S117.

20. Gariballa SE, Hutchin TP, Sinclair AJ. Antioxidant capacity after acute ischaemic stroke. QJM. 2002;95(10):685-690.

21. Rice-Evans C, Miller NJ. Total antioxidant status in plasma and body fluids. Methods Enzymol. 1994;234:279-293. 
22. Saini R. Ozone therapy in dentistry: a strategic review. J Nat Sci Biol Med. 2011;2(2):151-153.

23. Stübinger S, Sader R, Filippi A. The use of ozone in dentistry and maxillofacial surgery: a review. Quintessence Int. 2006;37(5):353-359.

24. Bocci V, Borrelli E, Travagli V, Zanardi I. The ozone paradox: ozone is a strong oxidant as well as a medical drug. Med Res Rev. 2009;29(4):646-682.

25. Lapchak PA, Araujo DM, Song D, Zivin JA. Neuroprotection by the selective cyclooxygenase-2 inhibitor SC-236 results in improvements in behavioral deficits induced by reversible spinal cord ischemia. Stroke. 2001;32(5):1220-1225.

26. Basso DM, Beattie MS, Bresnahan JC. A sensitive and reliable locomotor rating scale for open field testing in rats. $J$ Neurotrauma. 1995;12(1):1-21.

27. Cheng H, Cao Y, Olson L. Spinal cord repair in adult paraplegic rats: partial restoration of hind limb function. Science. 1996;273(5274):510-513.

28. Anderson DK, Means ED, Waters TR, Green ES. Microvascular perfusion and metabolism in injured spinal cord after methylprednisolone treatment. J Neurosurg. 1982;56(1):106113.

29. Oyinbo CA. Secondary injury mechanisms in traumatic spinal cord injury: a nugget of this multiply cascade. Acta Neurobiol Exp (Warsz). 2011;71(2):281-299.

30. Bocci V, Larini A, Micheli V. Restoration of normoxia by ozone therapy may control neoplastic growth: a review and a working hypothesis. J Altern Complement Med. 2005;11(2):257-265.

31. Viebahn-Hänsler R, León Fernández OS, Fahmy Z. Ozone in medicine: the low-dose ozone concept-guidelines and treatment strategies. Ozone Sci Eng. 2012;34:408-424.

32. Yamanel L, Kaldirim U, Oztas Y, et al. Ozone therapy and hyperbaric oxygen treatment in lung injury in septic rats. Int J Med Sci. 2011;8(1):48-55.

33. Uysal B, Demirbag S, Poyrazoglu Y, et al. Medical ozone therapy decreases postoperative uterine adhesion formation in rats. Arch Gynecol Obstet. 2012;286(5):1201-1207.

34. Sayın M, Temiz P, Var A, Temiz C. The dose-dependent neuroprotective effect of alpha-lipoic acid in experimental spinal cord injury. Neurol Neurochir Pol. 2013;47(4):345-351.

35. Kahveci R, Gökçe EC, Gürer B, et al. Neuroprotective effects of rosuvastatin against traumatic spinal cord injury in rats. Eur J Pharmacol. 2014;741:45-54.
36. Celik H, Karatay M, Erdem Y, et al. The biochemical, histopathological and clinical comparison of the neuroprotective effects of subcutaneous adalimumab and intravenous methylprednisolone in an experimental compressive spinal cord trauma model. Turk Neurosurg. 2016;26(4):622-631.

37. Aras M, Urfalı B, Serarslan Y, et al. Protective effects of minocycline against short-term ischemia-reperfusion injury in rat brain. Pediatr Neurosurg. 2013;49(3):172-178.

38. Huang C, Han X, Li X, et al. Critical role of connexin 43 in secondary expansion of traumatic spinal cord injury. $\mathrm{J} \mathrm{Neu}$ rosci. 2012;32(10):3333-3338.

39. Zhou KL, Chen DH, Jin HM, et al. Effects of calcitriol on experimental spinal cord injury in rats. Spinal Cord. 2016;54(7):510-516.

\section{Disclosures}

The authors report no conflict of interest concerning the materials or methods used in this study or the findings specified in this paper.

\section{Author Contributions}

Conception and design: Gürkan. Acquisition of data: Gürkan, Sayin, Kizmazoglu, Erdogan. Analysis and interpretation of data: Gürkan. Drafting the article: Gürkan, Yuceer. Reviewed submitted version of manuscript: Gürkan, Uzunoglu. Statistical analysis: Kizmazoglu. Administrative/technical/material support: Sayin, Erdogan, Yigitturk, Erbak Yilmaz. Study supervision: Sayin, Kaya, Yuceer.

\section{Supplemental Information \\ Previous Presentations}

This research was reported as an oral presentation at the 2018 Congress of the European Association of Neurosurgical Societies (EANS), August 2018, Brussels, Belgium.

\section{Correspondence}

Gokhan Gürkan: Katip Celebi University Atatürk Training and Research Hospital, Izmir, Turkey. gokhangurkan88@gmail.com. 